\title{
Recurrent groin hernia
}

\author{
P. J. CoX \\ B.Sc., M.B. B.S., F.R.C.S.
}

R. D. LEACH

M.B. B.S., F.R.C.S.

\author{
HAROLD ELLIS \\ D.M., M.Ch., F.R.C.S.
}

Surgical Unit, Westminster Hospital, London SWI

\begin{abstract}
Summary
One hundred consecutive recurrences following repair of inguinal hernias have been studied; 62 were direct, 30 indirect, 7 pantaloon and one a femoral hernia. Half the indirect recurrences occurred within a year of repair and probably represented failure to detect a small indirect sac. Later indirect recurrences probably represented failure to repair the internal ring. Nine of the direct hernias were medial funicular recurrences and represented failure to anchor the darn medially. The rest of the direct recurrences were attributable to tissue insufficiency and could probably have been averted by larger tissue bites.
\end{abstract}

Recurrences following inguinal herniorrhaphy remain an all too common problem but can be reduced by meticulous surgical technique.

\section{Introduction}

Inguinal hernias occur in some $3-4 \%$ of the population and account for approximately $10 \%$ of all general surgical operations (Zimmerman and Anson, 1967). Unfortunately, recurrences following repair are still all too common and amount to between 1 and $14.3 \%$ of published series (Moloney, 1958; Leading Article, 1977; Shuttleworth and Davies, 1960). Although $75 \%$ of recurrences occur within 3 years of the initial repair, recurrence can still take place 10 or more years after the initial operation (Shuttleworth and Davies, 1960) so that only a long-term follow-up can give the true picture of the success or failure of a particular technique of repair.

Any attempt at reduction of this recurrence rate must be based on a detailed knowledge of the mechanisms responsible for this complication. For this reason the authors investigated the surgical anatomy of 100 consecutive repairs of recurrent inguinal hernias and they also noted the relationship of the type of recurrence to the time from the initial repair.

\section{Methods}

All groin hernias operated upon at the Westminster Hospital Surgical Unit between 1972 and $N$ 1980 were reviewed. The type of hernia, the age at first presentation, the side, the length of hospital stay and whether or not the hernia was recurrent $z$ were noted. The search through the records was stopped when 100 recurrences which had undergone $\overline{3}$ surgery had been found.

\section{Results}

During this 8-year period, 890 hernias were repaired in 835 patients ( 55 were bilateral). One hundred of these hernias were recurrent. The type and the distribution of the hernias are shown in Table 1.

TABLE 1. Type and distribution of hernias.

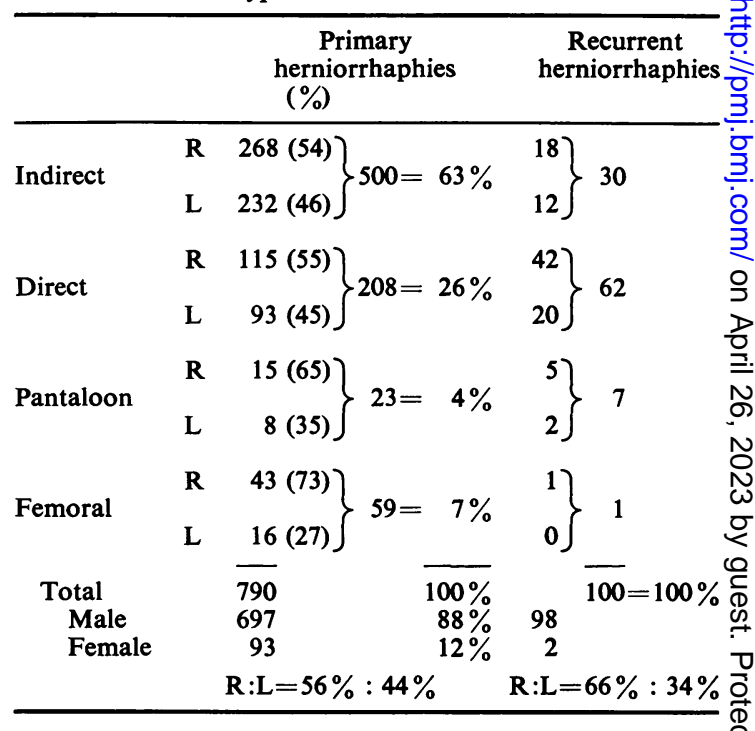


The mean age of all primary herniorrhaphies was 43 years compared with a mean age of 57 years at the time of repair of the recurrence. Only $2 \%$ of recurrences were in females, compared with $12 \%$ of the primary repairs. Of the 100 recurrences, 45 had been repaired initially at the Westminster Hospital.

\section{Anatomy}

Of the 790 primary herniorrhaphies, $56 \%$ were on the right side and $44 \%$ on the left side compared with $66 \%$ of the recurrences on the right side and $34 \%$ of the recurrences on the left side. Bilateral primary herniorrhaphies were performed in $55(7 \%$ of these patients) compared to $13 \%$ of the recurrences which had an initial bilateral inguinal hernia repaired. There was no case of simultaneous bilateral recurrence.

Of the 100 repairs of recurrent inguinal hernias, 62 were direct (53 of which were due to posterior wall weakness and 9 were medial funicular recurrences). Indirect recurrences occurred in 30 patients and there were 7 pantaloon hernias and one femoral hernia.

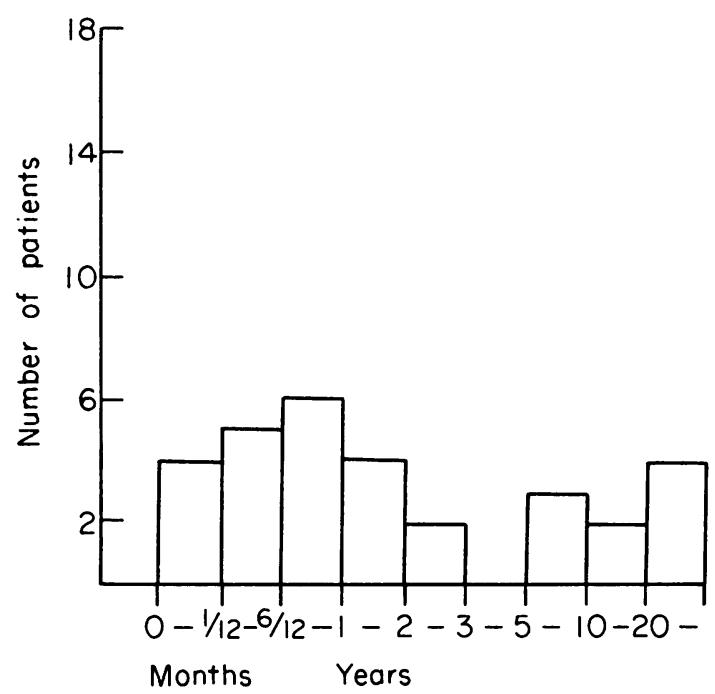

FIG. 1. Timing of indirect recurrence of 30 hernias.

\section{Time of recurrence}

Fifteen of the 30 indirect hernias were noted within one year and 4 within one month of the primary herniorrhaphy (Fig. 1). Of these immediate indirect recurrences, 3 were initially bilateral repairs of direct sacs elsewhere, the other being an indirect hernia repair which had been performed at the Westminster. The other 15 indirect recurrences occurred between one and 23 years after the primary herniorrhaphy. Only one direct recurrence occurred in the first month after operation, this was a medial

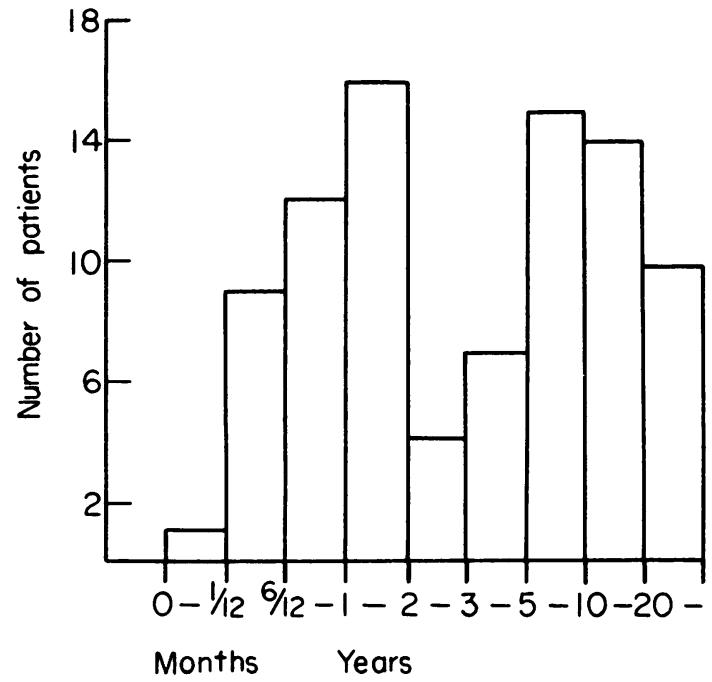

FIG. 2. Timing of direct recurrence of 88 hernias.

funicular recurrence (Fig. 2). This had initially been an indirect hernia which had been repaired at this hospital by a senior house officer with excision and ligation of the sac and nylon darn. Twenty-two of the direct recurrences had occurred within one year of primary repair and a further 20 recurred between one and 3 years after the initial repair. The rest of the direct recurrences occurred any time after the initial repair up until 25 years, which was the longest duration between initial repair and recurrence in this series. Recurrence occurred for the first time in 75 , the second time in 22 , the third time in 2 and for the fourth time in one of the patients. Thus there had been 129 previous repairs on 100 patients who presented with recurrent hernia. Only 2 of the 25 patients who had had more than one recurrence had indirect sacs and these had occurred at 7 years and 14 years after the initial repair.

\section{Discussion}

Recurrence of inguinal hernias may be due either to tissue insufficiency or to an inadequate repair. Poor tissues of the conjoined tendon and the inguinal ligament may lead to failure of repair, however strong the material used. A direct bulge may be overlooked in dealing with an indirect sac 
and may result in a later direct 'recurrence'. A direct medial recurrence seen after darn procedures may occur if the initial stitch of the darn is not firmly anchored in the periosteum of the pubic tubercle- 9 of the recurrences reported in this paper were of this type and are probably due to technical error, as was the immediate direct recurrence which occurred within one month.

Indirect recurrences may be seen as a result of either failure to detect a small indirect sac at the initial operation or inadequate surgical constriction of the internal ring which leads to development of the new sac. The immediate indirect recurrence in this series must have been due to inadequate excision of the indirect sac as well as inadequate constriction. Of the 30 patients who developed indirect recurrences, $50 \%$ had occurred within one year of their primary herniorrhaphy and it is probable that those for which the authors do not have information have, in fact, had a sac missed. A later group of indirect recurrences occurred between one and 23 years after the initial repair and these may well be due to the production of a pulsion sac via a lax, inadequately repaired, internal inguinal ring.

In a large series of 3572 primary herniorrhaphies by 70 surgeons reported by Palumbo and Sharpe (1971), 37 recurred. Of these, 21 were indirect. Seventy-five per cent. of the indirect recurrences appeared after 3 years.

Kostianen (1978) reported on 37 recurrences in a series of 278 patients. Thirteen were indirect, 16 direct, 6 femoral and 2 pantaloon. He also noted 2 groups of indirect recurrences, one within the year and a second up to 3 years after the initial repair. That the indirect recurrence is due to a missed sac is difficult to prove but the biphasic distribution of recurrences in both Kostianen's series and the present one is highly suggestive that the early group are in this category. The present series also shows that indirect recurrences can occur many years after the initial repair and accurate recurrence rates depend on long-term studies.

Post-mortem and surgical data have shown that a patent processus vaginalis is present into adult life in $20 \%$ of men (Conner and Peacock, 1973). Simple ligation and excision of the indirect sac leads to a $5 \%$ recurrence rate (McVay and Chapp, 1958) and of 3 large series of recurrent hernias, $50 \%$ were noted to be indirect (Quillnan, 1969; Thieme, $1971 ; \frac{\mathbb{2}}{\mathrm{Q}}$ Halverson and McVay, 1970).

Reduction of the incidence of indirect recurrence $\vec{\nexists}$ may be achieved if meticulous dissection of the cord $\stackrel{\text { क }}{+}$ and the internal ring is performed at all primary $\bar{C}$ herniorrhaphies. The late indirect recurrences may $\frac{\overline{\bar{O}}}{\mathrm{~s}}$ also be prevented by careful repair of the internal ring which just allows enough room for the vessels $\varrho$ and cord to leave from the abdomen.

Direct recurrence was only rarely demonstrated $\overrightarrow{-}$ in this series at the medial end of the inguinal canal. A carefully anchored repair at the pubic tubercle $\vec{\omega}$

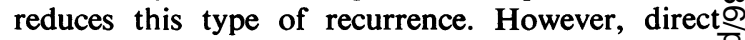
recurrence due to tissue insufficiency may not be soo readily prevented. In darn techniques, if sutures are 3 . placed too close to the edges of the posterior wallo defect these may cut out or the weak scar tissue that forms may subsequently stretch.

In closure of laparotomy wounds, large tissues bites with a long length of suture material and the ${ }^{N}$ placing of sutures close together may reduce the incidence of incisional hernia (Jenkins, 1980). $\overrightarrow{7}$ Application of these principles may further reduce $Z$ recurrence of direct inguinal hernias.

\section{References}

Conner. W.T. \& Peacock, E.E. (1973) Some studies on etiology of inguinal hernia. American Journal of Surgex, $\infty$ 126, 732.

Halverson, K. \& MCVay, C.B. (1970) Inguinal and femo hernioplasty. Archives of Surgery, 101, 127.

JENKINS, T.P.N (1980) Incisional hernia repair; a mechanical approach. British Journal of Surgery, 67, 335.

Kostianen, S. (1978) Concomitant groin hernia. Annales chirurgiae et gynaecologiae, 67, 214.

LEADING ARTICLE (1977) Activity and recurrent hernia. British Medical Journal, 2, 3.

MCVAY, C.B. \& CHAPP, J.D. (1958) Inguinal and femoral

hernioplasty. Annals of Surgery, 148, 499.
Moloney, G.E. (1958) Results of nylon darn repairs of hernia. Lancet, i, 273.

Palumbo, L.T. \& Sharpe, W.S. (1971) Primary inguinalo hernioplasty in the adult. Surgical Clinics of North America 51, 6, 1293.

QUILLNAN, R.H. (1969) Repair of recurrent inguinal hernia. 근 American Journal of Surgery, 118, 593.

ShutTleworth, K.E.D. \& Davies, W.H. (1960) Treatment윽 of inguinal herniae. Lancet, i, 126.

Thieme, E.T. (1971) Recurrent inguinal hernia. Archives of Surgery, 103, 238.

Zimmerman, L.M. \& ANSON, B.J. (1967) Anatomy and Surgery of Hernia, 2nd edn. Williams and Wilkins, Baltimore. 